\title{
Erratum to: Voluntary resistance wheel exercise from mid-life prevents sarcopenia and increases markers of mitochondrial function and autophagy in muscles of old male and female C57BL/6J mice
}

Zoe White ${ }^{1,2}$, Jessica Terrill ${ }^{1,3}$, Robert B. White ${ }^{1}$, Christopher McMahon ${ }^{4}$, Phillip Sheard ${ }^{5}$, Miranda D. Grounds ${ }^{1 *}$ and Tea Shavlakadze ${ }^{1}$

\section{Erratum}

Following publication of the original article [1] it was brought to our attention that there was a problem with the merging of the lines in Figs. 6 and 7. These figures show western blot images and each image used to have lines indicating separate groups. During production these lines merged into one single line and now the separate groups cannot be identified. The original article has since been corrected. Please see below for the corrected images:

\footnotetext{
Author details

'School of Anatomy, Physiology and Human Biology, The University of Western Australia (UWA), 35 Stirling Highway, Crawley, WA 6009, Australia. ${ }^{2}$ Centre for Cell Therapy and Regenerative Medicine, School of Medicine and Pharmacology, UWA and Harry Perkins Institute of Medical Research, Crawley 6009, WA, Australia. ${ }^{3}$ School of Chemistry and Biochemistry, UWA, Crawley 6009, WA, Australia. ${ }^{4}$ Developmental Biology Group, AgResearch Ltd, Hamilton 3214, New Zealand. ${ }^{5}$ Department of Physiology, University of Otago, Dunedin 9010, New Zealand.
}

Received: 4 January 2017 Accepted: 4 January 2017

Published online: 15 February 2017

\section{Reference}

1. White, et al. Voluntary resistance wheel exercise from mid-life prevents sarcopenia and increases markers of mitochondrial function and autophagy in muscles of old male and female C57BL/6J mice. Skeletal Muscle. 2016;6:45.

\footnotetext{
*Correspondence: miranda.grounds@uwa.edu.au

${ }^{1}$ School of Anatomy, Physiology and Human Biology, The University of

Western Australia (UWA), 35 Stirling Highway, Crawley, WA 6009, Australia
} 


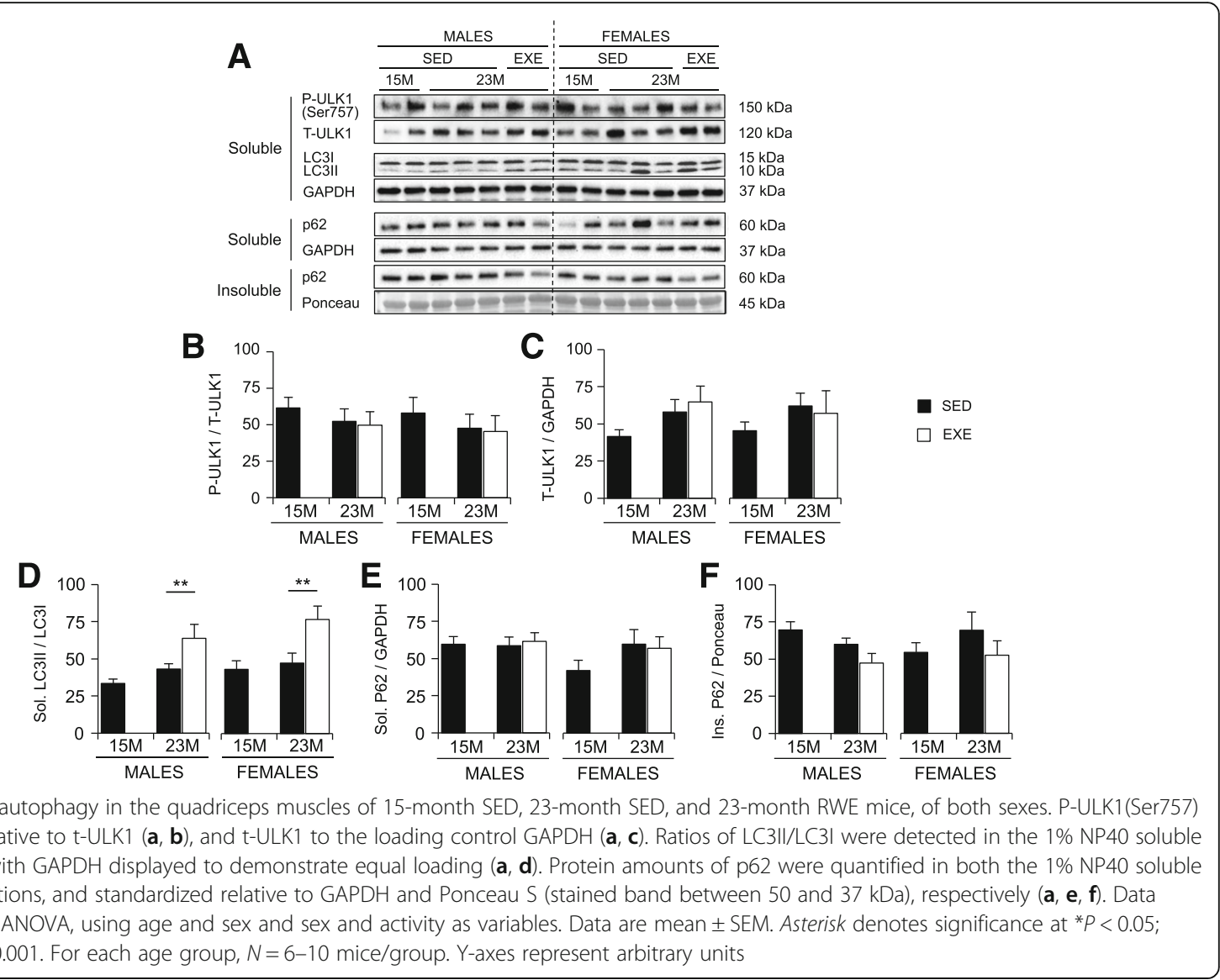




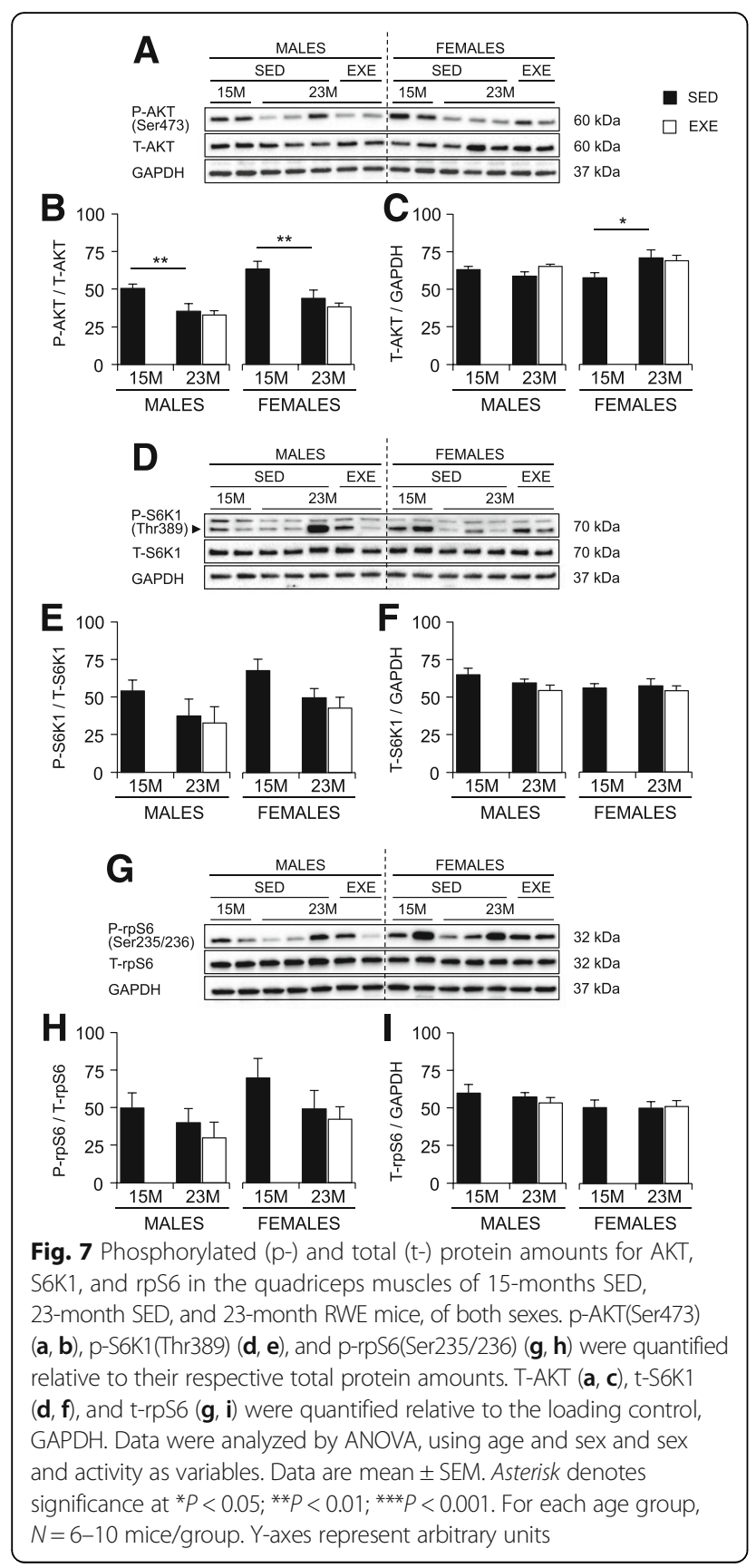

